\title{
Carnets
}

Revue électronique d'études françaises de l'APEF

Première Série - 2 Numéro Spécial 10-11 | 2011

D'un Nobel l'autre

\section{D'un discours l'autre : Mise en contexte littéraire et culturelle des discours de Stockholm de Cl. Simon et J-M G. Le Clézio}

José Domingues de Almeida

\section{OpenEdition}

Édition électronique

URL : http://journals.openedition.org/carnets/5421

DOI : 10.4000/carnets.5421

ISSN : 1646-7698

Éditeur

APEF

Édition imprimée

Date de publication : 1 janvier 2011

Pagination : 41-49

Référence électronique

José Domingues de Almeida, «D'un discours l'autre : Mise en contexte littéraire et culturelle des discours de Stockholm de Cl. Simon et J-M G. Le Clézio », Carnets [En ligne], Première Série - 2 Numéro Spécial 10-11 | 2011, mis en ligne le 16 juin 2018, consulté le 02 mai 2019. URL : http:// journals.openedition.org/carnets/5421; DOI : 10.4000/carnets.5421

\section{(c) (i) \&}

Carnets est mis à disposition selon les termes de la licence Creative Commons - Atribution - Pas d'utilisation commerciale 4.0 International. 


\title{
D'UN DISCOURS L'AUTRE.
}

\section{Mise en contexte littéraire et culturelle des discours de Stockholm de Cl. Simon et J-M G. Le Clézio ${ }^{1}$}

\author{
JOSÉ DOMINGUES DE ALMEIDA \\ Universidade do Porto - ILC Margarida Losa \\ jalmeida@letras.up.pt
}

\begin{abstract}
Résumé
Une (re)lecture comparative des deux discours prononcés à Stockholm par les deux lauréats français du Nobel de la Littérature, Cl. Simon et J-M. G. Le Clézio, fait apparaître des contextes littéraires et culturels diamétralement opposés dans l'approche même de la fiction narrative, qu'il y a tout lieu de creuser pour mieux mesurer la distance et les mutations en cours depuis quelque vingt ans dans le champ de la production littéraire en français.

Nous convoquerons, pour ce faire, les considérations tenues dans ces deux discours ; lesquelles portent un éclairage implicite à la caractérisation des enjeux liés à l'écriture française et francophone depuis deux décennies, et qui signalent un point de bascule dans la représentation que la littérature française se fait d'elle-même ou dans l'image que l'on s'en fait ailleurs.
\end{abstract}

\begin{abstract}
Comparative re-reading of both Cl. Simon and J-M. G. Le Clézio's speeches at Stockholm Nobel prize reward ceremony makes it clear that there is a diametrically opposed approach of narrative fiction between them which allows us to measure the distance and mutation in process within French and francophone literary field for about twenty years. It also shows a changing point as far as the way France sees itself or is seen elsewhere is concerned.
\end{abstract}

Mots-clés: Simon, Le Clézio, Littératrure, prix Nobel, contemporain, France Keywords: Simon, Le Clézio, Literature, Nobel Prize, contemporary, France

\footnotetext{
${ }^{1}$ Cette communication a été élaborée dans le cadre du projet "Interidentidades" de L'Institut de Literatura Comparada Margarida Losa de la Faculté des Lettres de l'Université de Porto, une I\&D subventionnée par la Fundação para a Ciência e a Tecnologia, intégrée dans le "Programa Operacional Ciência, Tecnologia e Inovação (POCTI)“, Quadro de Apoio III (POCTI-SFA-18-500).
} 
D’un Prix Nobel de la Littérature décerné à un écrivain français l'autre, les discours prononcés lors des cérémonies officielles trahissent ou congédient des tendances esthétiques, et expriment des soucis culturels, voire politiques, changeants, et dès lors très révélateurs de l'image que la France se fait de sa (la) littérature, et que l'on s'en fait partout ailleurs.

Une (re)lecture comparative des deux discours prononcés à Stockholm par les deux lauréats français du Nobel de Littérature, Cl. Simon et J-M. G. Le Clézio fait apparaître des contextes littéraires et culturels diamétralement opposés dans l'approche même de la fiction narrative, qu'il y a tout lieu de creuser pour mieux mesurer la distance et les mutations en cours depuis quelque vingt ans dans le champ de la production littéraire en français.

Elle signale également une évolution quant à la substance de l'argumentaire théorique appliqué à l'utilité ou fonction de l'activité littéraire; production, réception et système confondus, et cautionne l'idée d'une quête infinie, et cent fois réélaborée, des raisons de l'écriture.

Le Prix Nobel de Littérature décerné en 1985 au nouveau romancier Claude Simon marquait une reconnaissance et symbolisait un retrait. Une reconnaissance, dans le sens où Laurent Demoulin a interprété cet hommage: à savoir l'affirmation, à un moment où la littérature française s'engageait sur d'autres voies, et où le lauréat lui-même infléchissait son écriture à la faveur d'une tentative autobiographique, de l'incontournable supériorité et victoire de la Modernité littéraire sur l'Académie et la Tradition:

Les Modernes, finalement, sont sortis vainqueurs de la querelle que leur cherchaient les Anciens. Non seulement ils ont obtenu la reconnaissance publique (s'il est trop peu lu, Rimbaud est tout de même plus célèbre qu'Albert Samain et, en 1985, le prix Nobel a couronné Claude Simon et non Henri Troyat), mais surtout ils ont réussi à mener à terme leur projet (Demoulin, 1997: 8).

D'une certaine façon, cette attribution du Prix Nobel de Littérature à l'auteur de L'Acacia représente une charnière dans les représentations et les discours de la littérature française sur elle-même et coïncide avec l'expression d'un renouveau pensé par le biais des figures de la rétractation, de l'infléchissement et du retour (régression), avant d'assumer le "dépli" du texte sur le monde après son "repli", comme l'a souligné Sémir Badir (1993); un renouveau auquel les nouveaux romanciers ne sont guère étrangers.

En effet, au moment même où Claude Simon fait l'éloge de la modernité, des auteurs ayant considérablement contribué au "travail de la langue" et à l'intransitivité de l'écriture, amorcent un mouvement évolutif, de "dépli" (auto)référentiel, d'ouverture sur la représentation, le monde et le sujet; ou bien renient carrément leurs assurances modernes 
de naguère.

Ainsi, la critique souligne les signes de tarissement d'une certaine conception intransitive et "déconstructive" de l'écriture romanesque ou, autrement dit, un point de nonretour dans l'aventure textuelle d'où les écrivains ne pourraient sortir que par un "dépli" et un compromis avec la représentation, au risque d'une moins-value esthétique et hermétique au moment de leur parution (Demoulin, 1997: 8).

Demoulin constatera que "la logique du comble est arrivé à un point de non-retour" (ibidem), et Badir (1999: 254), avec Compagnon, que "le Bourgeois ne se laisse plus épater" (1990: 7) et qu'un seuil critique a été atteint où le jeu d'un improbable effet esthétique ne vaut plus la chandelle du travail de surinvestissement intransitif.

II s'agit d'Eden, Eden, Eden de Pierre Guyotat, Paysages de ruines avec personnages de Danièle Sallenave et Louve basse de Denis Roche, que Bruno Blanckeman qualifie de "derniers des grands 'mécrits' textuels" (Blanckeman, 2000: 13s.).

De même, en 1975, les milieux littéraires parisiens s'étonnent de voir l'auteur de $L e$ Degré zéro de l'écriture passer aux confidences, à l'autocritique et à l'anecdote, et s'adonner au "plaisir" du texte. L'heure est aux affirmations hypothétiques, aux tâtonnements théoriques comme en témoigne cette notion tardive du texte recevable, "texte ardent" que Roland Barthes place à côté du texte lisible et du texte scriptible (Barthes, 1976: 143).

En 1983, c'est au tour de Philippe Sollers de se rétracter et de choisir le genre romanesque. Femmes, roman davantage "figuratif", entérine le passage de Sollers de Tel Quel à L'Infini et confirme un tournant à 180 degrés vers le récit et "la vérité romanesque" (Hollier, 1993: 1021).

Denis Hollier livre un commentaire métaphorique de ce roman par lequel, une fois franchies les limites, Sollers renouerait avec le plaisir de la fiction. Selon lui, Femmes mettrait en fiction l'"embarcation de la fiction même" (idem, 1022) vers une contrée improbable; "une percée hors de la finitude française" (ibidem); celle d'une période et d'une époque littéraires qu'il s'agit de congédier à présent.

En 1984, Alain Robbe-Grillet publie un texte qui, au dire de son auteur, mûrissait depuis quelques années. L'heure, il est vrai, est aux rétractations, aux mises à jour et aux bilans. Le nouveau romancier n'y échappe pas, étant donné "la soumission moqueuse [de l'écriture] aux préoccupations du moment" (Robbe-Grillet, 1984: 9).

Dans la foulée des "retours à", Robbe-Grillet insinue ne prétendre qu'à une "relève" (ibidem), mais, venant de lui, le mot en dit long sur la mutation en cours. Puis vint cet aveu, que d'aucuns liront comme une trahison: “Je n'ai jamais parlé d'autre chose que de moi. Comme c'était de l'intérieur, on ne s'en est guère aperçu" (idem, 10). Cet aveu contrariait trois décennies hostiles au moi, à la psychologie du personnage et à la représentation.

Le fait qu'Alain Robbe-Grillet et d'autres nouveaux romanciers passent à 
l'autobiographie de façon tout aussi surprenante atteste le bien fondé des propos de JeanPierre Salgas. La tentation autobiographique et confidentielle correspond bel et bien à "ce qui reste quand tout a été déconstruit: l'auteur et ses masques" (Salgas, 1997: 14).

L'appropriation postmoderne de Le miroir qui revient ne manquera pas d'y lire une oscillation ironique entre le dire et le dédire; un travail, en défiance, des données autobiographiques, du vécu et du regard jeté en amont (cf. Van Montfrans, 1986: 81-89) qui fait inévitablement en sorte que, à son tour, le lecteur soit légitimement tenté de "relire" autrement la production littéraire du Nouveau Roman, et la voie dotée "d'un sujet", en déstructuration (Varga, 1986: 11).

On a affaire à un retrait, si l'on considère les métamorphoses et le ressourcement de l'écriture et de la modernité à partir des années quatre-vingt, et l'émergence d'autres catégories, bien que contiguës, vis-à-vis de l'héritage littéraire moderne, que ce soit le roman minimaliste, l'autofiction ou toutes les modalités postmodernes d'approche du récit.

Dans ce contexte, Le discours de Stockholm de Cl. Simon (1986) entend non pas dresser le bilan de l'état des lieux en regard du passé, mais plutôt, profitant de la solennité de l'occasion, solder des comptes avec l'Académisme et l'Engagement. Implicitement, toutefois, le discours tient déjà compte de la nouvelle donne fictionnelle qui s'installe à ce moment. Le roman réaliste de type balzacien se voit directement visé par le discours (idem, 15) au profit de l'“art pour l'art" (idem, 11), de l'intransitivité de l'écriture et du souci ricardolien de produire plutôt du sens que d'en exprimer (cf. idem, 21); à savoir le refus de toute causalité extrinsèque au fait littéraire.

Suivant un raisonnement dont les théoriciens du postmoderne littéraire se serviront pour encadrer la contiguïté et la réinterprétation des rapports de l'écriture au réel, $\mathrm{Cl}$. Simon insiste sur le filtrage scriptural de la réalité: “(...) ces récits n’ont d'autre réalité que celle de l'écriture qui les instaure" (idem, 29).

De ce point de vue, le lauréat affiche une posture indépendante à l'égard de ceux qui voudraient accaparer son œuvre à des fins politiques (cf. idem, 11). La psychologie des personnages n'est pas radicalement niée, mais ses moyens demeurent l'objet d'un soupçon fondé sur une théorie littéraire; ce qui nous ramène aux prémisses de la littérature française contemporaine (cf. idem, 15 \& 26-28).

Mais la littérature situationnelle de Jean-Paul Sartre, autre lauréat français du prix Nobel, n'est pas épargnée non plus. Bien au contraire, Simon réfute l'idée d'un savoir préalable dispensé par l'écrivain au lecteur où il voit la continuation, sous une autre forme, de l'instruction religieuse, "celle de la parole, de la fable" (idem, 14). Et Simon d'aller plus loin dans sa diatribe: "D'ailleurs, s'il m'avait été révélée quelque vérité importante dans l'ordre social, de l'histoire ou du sacré, il m'eût semblé ridicule d'avoir recours pour l'exposer à une fiction inventée au lieu d'un traité raisonné de philosophie, de sociologie ou de théologie" 
(idem, 24).

L'incontournable question "Qu'est-ce que la littérature?" ou celle, tout aussi entachée d'humanisme et d'engagement intellectuel, "Que peut la littérature?", se voient renvoyées dos à dos au profit d'une persévérance du travail intransitif de l'écriture.

Peine qui n'est pas tout à fait perdue si l'on considère les enjeux de la fiction contemporaine, quoique différemment placée, elle aussi, sous l'emprise d'une suspicion. Comme Jean-Pierre Salgas l'a bien résumé, Le Discours de Stockholm plaide la cause, entendue, il est vrai, du triomphe de l'opacité de l'écriture et de la technique face à la transparence ou au message (cf. Salgas, 1997: 11).

À cet égard, du fait de son évolution scripturale propre et de la teneur de son discours prononcé à Stockholm en 2008, J-M. G. Le Clézio incarne le point de bascule esthétique et théorique qui a caractérisé la littérature française dans le tournant des années quatre-vingt et l'approche élargie et enrichie des littératures dites francophones.

L'évolution opérée dans les prémisses et les soucis de l'écriture entre l'auteur du Procès verbal et celui de La Ritournelle de la faim, plus explicitement depuis Désert, ainsi que les propos du discours de Le Clézio, signalent une réflexion et une pratique de l'écriture en rupture avec l'héritage textuel des générations antérieures.

À cet égard, force est de constater que la question du pouvoir de la littérature hante toujours les esprits quelque vingt ans plus tard, même si il n'est plus dans l'air du temps de la poser de la même façon, ou surtout pas avec les mêmes enjeux. En fondant le besoin de l'écriture sur son vécu personnel en contexte difficile, les contraintes de la guerre, et sur ce rapport de curiosité au Monde: "regarder la vie extérieure par le carré de sa fenêtre" (2008: 2), Le Clézio place la conception du fait littéraire au-delà des approches existentielle ou intransitive pour y voir, à la faveur de ce Dagerman qui influença son univers enfantin, un acte marqué par l'ambiguïté, la "forêt de paradoxes" (ibidem): "C'est la pensée de Dagerman qui m’envahit plutôt que le constat militant ou le pari désabusé de Sartre" (idem, 3).

Le moment n'est plus aux théories hermétiques, ni aux engagements révolutionnaires: "L'écrivain (...) n'a plus l'outrecuidance de croire qu'il va changer le monde (...)“ (idem, 4). L'heure est plutôt de s'interroger sur les apories de l'écriture dans un monde lui-même marqué par les contradictions de la mondialisation. En tous cas, une chose est sûre pour l'écrivain d'aujourd'hui, selon Le Clézio: "Ce n'est pas toujours un séjour agréable. Lui qui se croyait à l'abri, elle qui se confiait à sa page comme à une amie intime et indulgente, les voici confrontés au réel (...)" (idem, 3). Et les soucis de la littérature consistent justement à l'expression de tous ces paradoxes.

Ce faisant, l'écrivain se veut "témoin malgré lui" (idem, 5); ce qui le place au centre d'une terrible et douloureuse contradiction: "Agir, c'est ce que l'écrivain voudrait par-dessus tout. Agir, plutôt que témoigner. Ecrire, imaginer, rêver, pour que ses mots, ses inventions et 
ses rêves interviennent dans la réalité, changent les esprits et les cœurs, ouvrent un monde meilleur" (idem, 5), qui le rendent encore plus conscient de "sa propre incapacité" (ibidem).

Entre travail scriptural et souci du monde, mutation que l'évolution esthétique de Le Clézio acte ou inaugure, c'est selon, ce sont les dimensions et horizons nouveaux de la littérature en langue française qui se trouvent affectés ou convoqués dans un débat sur son utilité, son attractivité et ses modalités de rayonnement.

Plus que jamais, même si "la crise du roman" est concomitante au roman lui-même comme le rappelle Dominique Viart (1996), et surtout en France, "nation littéraire" s'il en est (Fergusson, 1991), la question du rapport au fait et à l'institution littéraires accuse les symptômes d'un malaise aux diagnostics contradictoires.

D'aucuns n'ont pas hésité à les imputer au travail scriptural outrancier de l'hermétisme parisien, à l'origine de la "sinistrose" (Domenach, 1995) ou du tarissement démocratique du genre romanesque (Raczymov, 1994) ou son fourvoiement commercial (Nadaud, 1992), mais d'autres ne démordent toujours pas de la radicalité moderne de l'écriture dont l'infléchissement serait à l'origine d'une dangereuse perte de "négativité" textuelle aux retombées politiques droitières (Prigent, 1991): un débat circulaire contribuant à ce que François Taillandier nomme la "délectation morose" (2009: 18).

"Pourquoi écrit-on?" hante toujours les esprits. Antoine Compagnon, dans un récent essai, a bien du mal à y répondre, et pour tout dire, tourne autour du pot causal avec un même manque d'assurance qui n'a de corolaire inverse que l'aversion que le pouvoir en place voue à l'utilité des Humanités. Plus de questions angoissées que de réponses convaincantes: "Si la question se pose, serait-ce parce qu'il est trop tard pour y répondre?" (Compagnon, 2007: 39).

Bien sûr, l'auteur de Les démons de la théorie passe en revue les atouts et atours de la littérature depuis deux siècles, qu'ils soient mimétiques, démocratiques ou esthétiques, avant de se centrer, après Samuel Johnson, sur un rôle moins "politique" ou "théorique" du fait littéraire, celui qui concerne une éthique de l'écriture et, partant, de la lecture: “(...) le texte littéraire me parle de moi et des autres" (idem, 65). Ce faisant, "la littérature déconcerte, dérange, déroute, dépayse plus que les discours philosophique, sociologique ou psychologique, parce qu'il fait appel aux émotions et à l'empathie" (idem, 67).

Oui, il est certes "des choses que seule la littérature peut nous donner" (idem, 72), mais quoi? Comment les exprimer? Certes, la littérature est "cet exercice de pensée". Mais, la pensée de cet exercice n'aurait-il pas mis à mal, à la longue, son évidence et sa pertinence (cf. idem, 70)?

Ces questions, plus nombreuses que les réponses, pointent une "forêt paradoxale" qu'habitent Le Clézio et son discours de Stockholm. Si "la littérature est faite de langage" (Le Clézio, 2008:6), c'est lui qui assigne sa place à l'écrivain au service de cette donnée 
ontologique et anthropologique primordiale.

Dès lors, dans un contexte déséquilibré, mais simultanément mondialisé des cultures, l'écriture assume un rôle de médiation incontournable entre les différents états culturels, du mythe à l'érudition: "Il n'est pas possible de fonder le respect d'autrui et l'égalité sans donner à chaque enfant le bienfait de l'écriture" (idem, 7).

Ce faisant, Le Clézio fonde désormais l'approche de l'écriture non plus sur une perspective autotélique et hermétique moderne du texte, mais plutôt sur un horizon postmoderne ouvert aux complexités et perplexités du monde contemporain: "Aujourd'hui, au lendemain de la décolonisation, la littérature est un des moyens pour les hommes et les femmes de notre temps d'exprimer leur identité, de revendiquer leur droit à la parole, et d'être entendus dans leur diversité. Sans leur voix, sans leur appel, nous vivrions dans un monde silencieux" (ibidem).

Raison pour laquelle le lauréat n'oubliera pas d'invoquer, en si solennelle occasion, tous ceux et toutes celles qui, selon lui, et ils / elles sont légion, disent ou écrivent ce "quelque chose de simple, de vrai, qui n'existe que dans le langage" (idem, 10) et qui ressortit toujours du paradoxe.

Raison pour laquelle aussi cet écrivain franco-mauricien, mais que les nomenclatures en place n'ont jamais désigné de "francophone", est l'un des signataires du Manifeste 2006 anti-francophone, lui-même, à maints égards, paradoxal dans les termes.

Dans un certain sens, J-M G. Le Clézio incarne, de par son parcours romanesque personnel, les mutations actées par les souscripteurs dudit Manifeste dans leur renouement avec le réel et les bruissements du monde:

\footnotetext{
Le monde revient. Et c'est la meilleure des nouvelles. N'aura-t-il pas été longtemps le grand absent de la littérature française? Le monde, le sujet, le sens, l'histoire, le "référent": pendant des décennies ils auront été mis "entre parenthèses" par les maîtres penseurs, inventeurs d'une littérature sans autre objet qu'elle-même, faisant, comme il se disait alors "sa propre critique dans le mouvement même de son énonciation" (A.A.V.V., 2007).
}

En fait, ces auteurs, dont les témoignages devaient être repris dans l'ouvrage collectif que l'on sait (Le Bris \& Rouaud, 2007), exprimaient une impressionnante victoire de la langue française, quelle que soit la désignation qu'on lui attribue; une chance pour la "dénationalisation de la langue et des histoires" (Almeida, 2006), une issue pour l'“hypostase" (Almeida, 2008) dans laquelle l'Hexagone tenait l'aura de la fiction; un point de bascule décisif dans l'image entretenue: 
Nous vivions encore dans l'évidence de la France, de la littérature et de la langue françaises; c'était comme une vaste maison vénérable, avec des secrétaires emplis de papiers de famille, et, sur les murs, des portraits d'ancêtres, souvent en costume d'académiciens (Taillandier, 2009: 22).

Et si le français, contre toute attente, devenait une de ces langues et une de ces voix de cette mondialisation autant décriée que souhaitée, pour n'en pas finir avec les apories?

Alain Mabanckou, lauréat-monde d'un prix littéraire hexagonal, lève le voile de ce que peut ou doit être le concept par-delà les paradoxes, et désamorce une diatribe futile:

La littérature-monde en langue française est la reconnaissance et la prise de conscience de notre apport à l'intelligence humaine, avec cet outil qu'est la langue française, cet outil que beaucoup ont hérité de façon conflictuelle, d'autres par choix, d'autres encore parce que leurs ancêtres étaient les Gaulois - mais faut-il passer notre existence à accuser le passé ou à bâtir un avenir? Notre tâche est de suivre la marche de cette littérature-monde en langue française, de tracer sommairement ses contours, de la regarder dans un ensemble plus étendu, plus éclaté, plus bruyant, c'est-à-dire le monde (Le Bris \& Rouaud, 2007: 65). 


\section{Bibliographie}

A.A.V.V. (2007). "Pour une littérature-monde en français". In: Le Monde, 16 mars.

ALMEIDA, José Domingues de (2006). "Des avantages à lire comme autant d'inconvénients. La Francophonie face à ses enjeux". In: http://www.apef.org.pt/actas2006/JA122006.pdf, pp. 31-43.

ALMEIDA, José Domingues de (2008). "Le français, langue hypostasiée. Excursus littéraire et théorique. Relevé de quelques atouts, ambiguïtés et apories". In: Fátima Oliveira \& Isabel Duarte orgs. O Fascínio da Linguagem. Porto: Centro de Linguística da Universidade do Porto / FLUP, pp. $255-263$.

BADIR, Sémir (1993). "Vers la postmodernité. Retour à Baudelaire?”. In: Ecritures, no 5, pp. 8-21.

BADIR, Sémir (1999). "Histoire littéraire et postmodernité". In: Ecritures contemporaines, no 2, pp. 241264.

BARTHES, Roland (1976). Roland Barthes por Roland Barthes. Lisboa: Edições 70.

BLANCKEMAN, Bruno (2000). Les récits indécidables: Jean Echenoz, Hervé Guibert, Pascal Quignard. Paris: Presses Universitaires du Septentrion.

Compagnon, Antoine (1990). Les cinq paradoxes de la modernité. Paris: Seuil.

COMPAGNON, Antoine (2007). La littérature pour quoi faire?. Paris: Fayard.

DeMOULIN, Laurent (1997). "Génération innommable". In: Textyles, no 14, pp. 7-17.

DOMEMACH, Jean-Marie (1995). Le crépuscule de la culture française?. Paris: Plon.

FERGUSON, Priscilla Parkhurst (1991). La France, nation littéraire. Bruxelles: Labor.

HOLLIER, Denis (1993). "Comment peut-on être français?". In: Denis Hollier org. De la Littérature française. Paris: Bordas, pp. 1020-1027.

LE BRIS, Michel / RouAUd, Jean (2007). Pour une littérature-monde. Paris: Gallimard.

LE CLEZIO, Jean-Marie Gustave (2008). "Dans la forêt des paradoxes". In: http://nobelprize.org/nobel_prizes/literature/laureates/2008/clezio-lecture_fr.pdf (consulté le 27 décembre 2009), pp. 1-11.

NADAUD, Alain (1992). Malaise dans la littérature. Paris: Champ Vallon.

PRIGENT, Christian (1991). Ceux qui merdRent. Paris: P.O.L.

SALGAS, Jean-Pierre (1997). "1960-1990: romans mode d'emploi". In: Roman français contemporain. Paris: Ministère des Affaires Etrangères, pp. 7-52.

SIMON, Claude (1986). Discours de Stockholm. Paris: Minuit.

TAILLANDIER, François (2009). La langue française au défi. Paris: Flammarion.

RACZYMOW, Henri (1994). La mort du grand écrivain. Essai sur la fin de la littérature. Paris: Stock.

ROBBE-GRILLET, Alain (1984). Le miroir qui revient. Paris: Gallimard.

VAN MONTRFRANS, Manet (1986). "Vers une issue de l'impasse postmoderne". In: Littérature et postmoderrnité. Amsterdam / Atlanta: Rodopi, pp. 81-89.

VARGA, Aaron Kibédi (1986). "Récit et postmodernité". In: Littérature et postmodernité. Amsterdam / Atlanta: Rodopi, pp. 1-16.

VIART, Dominique (1996). “Le roman en question III”. In: Prétexte, no 11, pp. 63-68. 
